\title{
Editorial
}

\section{Recent Advances in Oscillation Theory 2011}

\author{
Yuri V. Rogovchenko, ${ }^{1}$ Leonid Berezansky, ${ }^{2}$ Elena Braverman, ${ }^{3}$ \\ and Josef Diblík 4 \\ ${ }^{1}$ Department of Mathematics and Mathematical Statistics, Umea University, 90187 Umeå, Sweden \\ ${ }^{2}$ Department of Mathematics, Ben-Gurion University of the Negev, Beer-Sheva 84105, Israel \\ ${ }^{3}$ Department of Mathematics and Statistics, University of Calgary, 2500 University Drive NW, \\ Calgary, AB, Canada T2N1N4 \\ ${ }^{4}$ Department of Mathematics, Faculty of Electrical Engineering and Computer Science, \\ Brno University of Technology, 61600 Brno, Czech Republic
}

Correspondence should be addressed to Yuri V. Rogovchenko, yuriy.rogovchenko@math.umu.se

Received 29 September 2011; Accepted 29 September 2011

Copyright (C) 2011 Yuri V. Rogovchenko et al. This is an open access article distributed under the Creative Commons Attribution License, which permits unrestricted use, distribution, and reproduction in any medium, provided the original work is properly cited.

Theory of oscillations is an important and well-established branch of the modern theory of differential equations concerned, in a broad sense, with the study of oscillatory phenomena arising in applied problems in technology, natural, and social sciences. Theoretical aspects of the classical theory of oscillations regard existence and nonexistence of oscillatory (periodic, almost periodic, etc.) solutions to a given equation or system, and description of asymptotic behavior of such solutions. It is well known that oscillation of solutions is an intrinsic feature of many dynamical systems. Furthermore, oscillations can be induced in a nonoscillatory system by nonlinear terms, delayed or advanced arguments, randomness, though these factors may also destroy oscillations arising in the original system.

Papers included in this special issue address a number of challenging problems related to nonlinear oscillations, and describe novel techniques and approaches to classical problems in the theory of oscillations and beyond. Although the main focus of this issue is on oscillations, the editors are pleased to acknowledge several interesting contributions that develop new general methods applicable to wide classes of problems and deal with questions that are not directly related to oscillations.

The issue opens with the contribution by H. A. Agwo et al. who use a generalized Riccati transformation, monotonicity arguments, and standard results on dynamic equations for establishing oscillation criteria for a class of second-order nonlinear delay dynamic equations on time scales. Several examples illustrate theoretical results.

V. G. Angelov and D. Tz. Angelova study a lossless transmission line terminated by a nonlinear resistive load and parallel connected capacitance. Using Krasnoselskii fixed point theorem, they establish existence of solutions to an equivalent initial value problem for 
a neutral functional differential equation with prescribed set of zeros on a half-axis. The paper concludes with a numerical example.

The work of K. Boukerrioua is concerned with a class of nonlinear integral inequalities that can be used for establishing global existence, uniqueness, stability, boundedness, and other properties of solutions to nonlinear differential equations. To illustrate possible applications, the author obtains bounds for solutions of two nonlinear differential equations in terms of coefficients of given differential equations.

In the paper by X. Chen and Y. Xia, a topological decoupling problem of two nonlinear differential equations is considered from the point of view of a generalized exponential dichotomy, a new concept that has been introduced in order to overcome restrictions on the dynamics imposed by the classical notion of exponential dichotomy. The main result in the paper establishes topological equivalence of two nonlinear differential equations, and, thus, a oneto-one correspondence between their solutions.

N. A. Khan, M. Jamil, S. A. Ali, and N. A. Khan deal with a new approximate method for solving a nonlinear Duffing-van der Pol equation describing, for instance, the dynamics of a point mass in a double-well potential or a periodically forced steel beam deflected toward two magnets. The authors employ a modified version of a homotopy perturbation method based on two components of a homotopy series. In order to improve the accuracy of the solution, they apply the Laplace transform and convert the transformed series into a meromorphic function by using Pade approximants. An analytic solution to the problem is obtained through the inverse Laplace transform, whereas the fourth-order Runge-Kutta algorithm is used for solving the governing equation. Two numerical examples are provided to demonstrate the validity of the proposed method; the influence of parameters on the accuracy of the method is investigated.

In the paper by N. A. Khan, M. Jamil, and A. Ara, a modification of He's Hamiltonian approach is suggested for establishing the natural frequency of a discontinuous nonlinear oscillator with the elastic force term proportional to the sign of displacement. A higher-order approximation to the solution of a nonlinear oscillator equation is constructed, and a high-accuracy approximate frequency-amplitude relationship is derived. Numerical results are provided to illustrate the efficiency of the suggested technique.

The contribution by A. Pascoletti and F. Zanolin that closes this special issue is motivated by the original approach to the celebrated Poincaré-Birkhoff twist theorem, also known as the Poincare last geometric theorem suggested, for the case of analytic functions, in a monograph of Professor Tongren Ding. The authors extend Ding's definition to a pure topological setting and obtain fixed point theorems for continuous bend-twist maps without requiring regularity properties of the maps involved. It is not assumed that the maps are homeomorphisms nor area-preserving property or invariance of the boundaries is supposed. Some results are stable also under small perturbations, which prompts possibilities for applications to nonconservative planar differential systems. The main existence theorem follows from the Borsuk separation theorem and Alexander's lemma. Partially extending Ding's theorem to the nonanalytic setting, the authors establish, contrary to the existence of two fixed points in Ding's result, existence of at least one fixed point. They demonstrate on a simple example that, in some situations, only one fixed point may actually occur. In the final part of the paper, an application of abstract results to a periodic problem for a class of nonlinear ordinary differential equations is outlined.

Seven papers published in this special issue represent only selected directions of research conducted nowadays in the theory of oscillations and related areas, featuring solutions to important theoretical and applied problems. Addressing different aspects of nonlinear 
oscillations for several important classes of ordinary, dynamic or functional differential equations, contributions included in this special issue emphasize the breadth of the research field and unveil interesting relationships between oscillation and other fundamental properties of solutions.

Recent progress in the Oscillation Theory of ordinary, functional, neutral, partial, impulsive differential equations, and their discrete versions has been attributed to an elevated interest to the topic that inspired and keeps inspiring many scholars. We hope that researchers will find in this special issue new ideas and techniques that will stimulate further progress in this important branch of the qualitative theory of differential equations and related areas.

Yuri V. Rogovchenko

Leonid Berezansky

Elena Braverman

Josef Diblík 


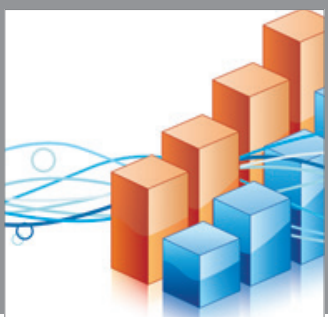

Advances in

Operations Research

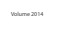

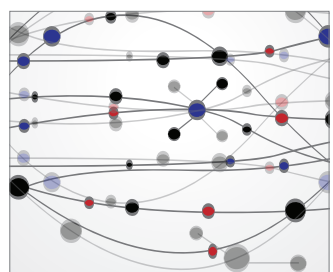

\section{The Scientific} World Journal
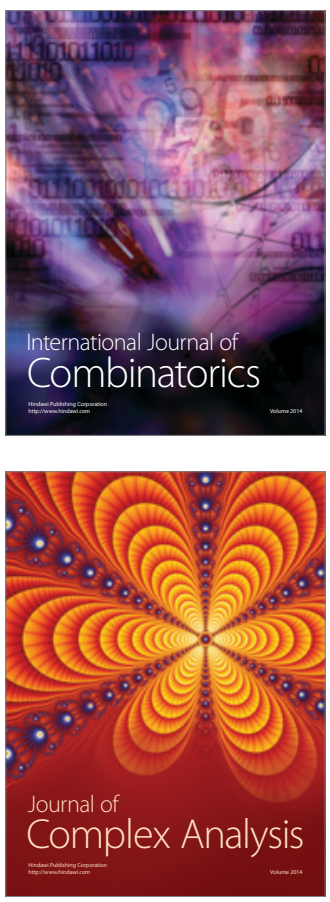

International Journal of

Mathematics and

Mathematical

Sciences
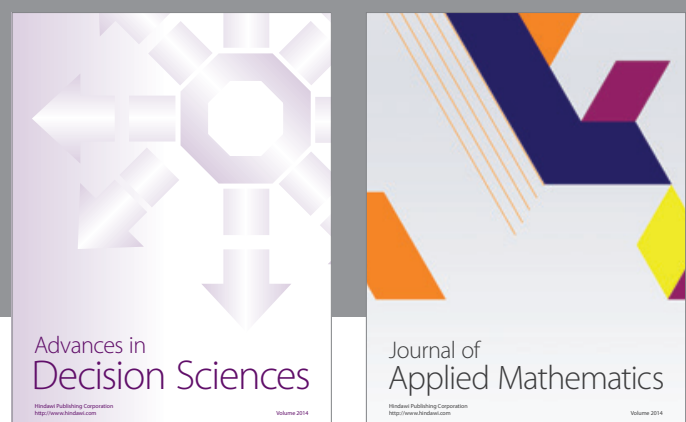

Journal of

Applied Mathematics
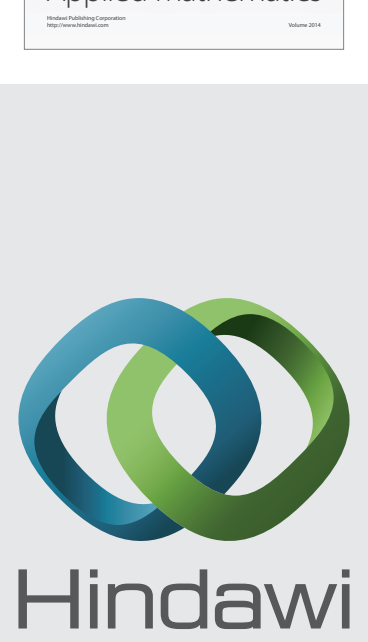

Submit your manuscripts at http://www.hindawi.com
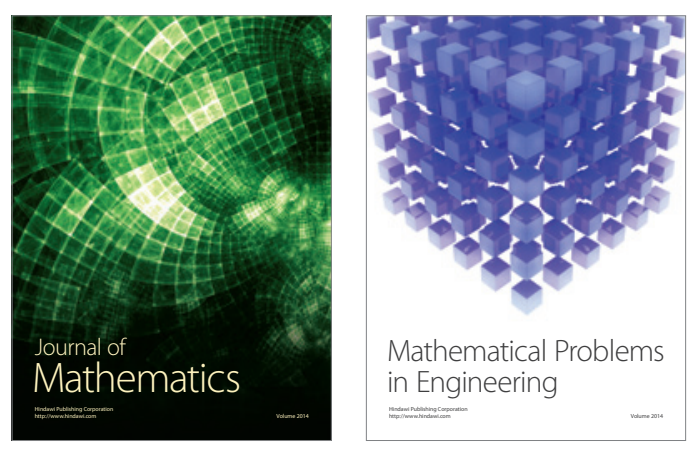

Mathematical Problems in Engineering
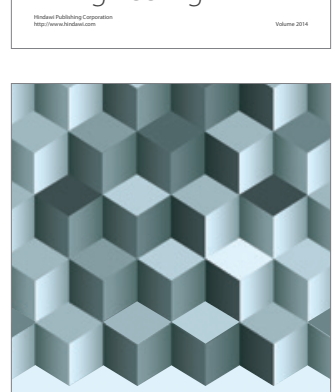

Journal of

Function Spaces
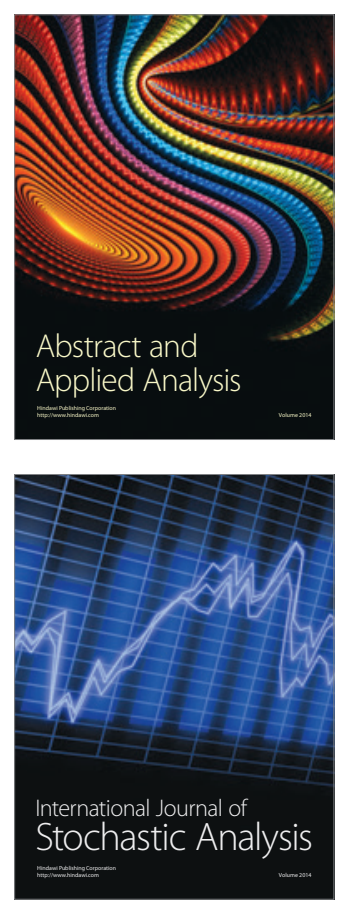

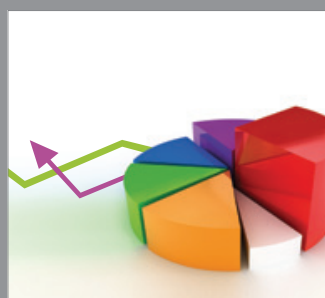

ournal of

Probability and Statistics

Promensencen
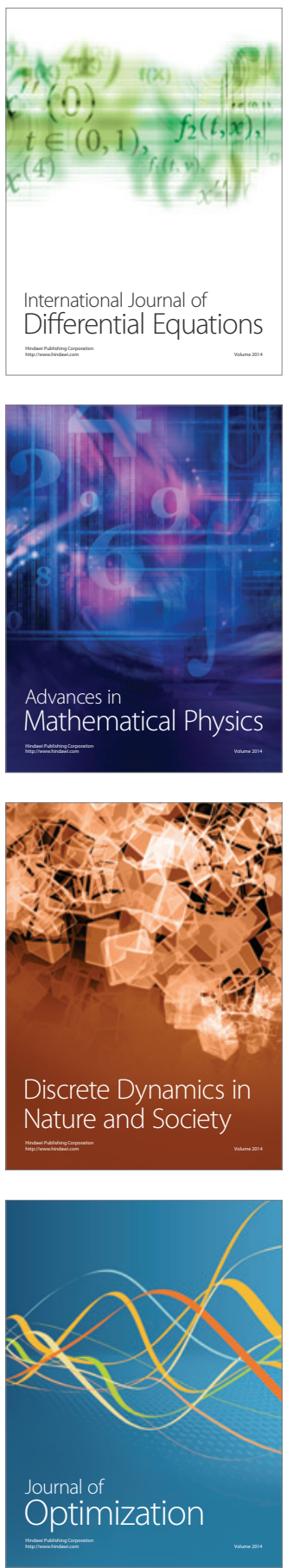